\title{
Efetividade de tecnologia educacional para prevenção de quedas em ambiente hospitalar
}

Effectiveness of educational technology for preventing falls in a hospital environment Efectividad de tecnología educativa para la prevención de caídas en el ambiente hospitalario

Maria Aline Moreira Ximenes ${ }^{1}$ id https://orcid.org/0000-0002-1674-3357 Maria Girlane Sousa Albuquerque Brandão² io https://orcid.org/0000-0002-9925-4750

Thamires Sales Macêdo ${ }^{3}$ id https://orcid.org/0000-0002-3896-0184

Mágila Maria Feijão da Costa ${ }^{3}$ id https://orcid.org/0000-0002-7705-6905

Nelson Miguel Galindo Neto ${ }^{4}$ (D https://orcid.org/0000-0002-7003-165X

Joselany Áfio Caetano ${ }^{1}$ id https://orcid.org/0000-0002-0807-056X

Mônica Oliveira Batista Oriáa ${ }^{1}$ ic https://orcid.org/0000-0002-1483-6656

Livia Moreira Barros² io https://orcid.org/0000-0002-0174-2255

Como citar:

Ximenes MA, Brandão MG, Macêdo TS, Costa MM, Galindo Neto NM, Caetano JA, et al. Efetividade de tecnologia educacional para prevenção de quedas em ambiente hospitalar. Acta Paul Enferm. 2022;35:eAPE01372.

DOI

http://dx.doi.org/10.37689/acta-ape/2022A001372

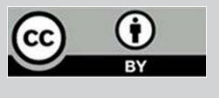

Descritores

Educação em saúde; Acidentes por quedas; Prevenção de acidentes; Tecnologia educacional; Hospitais

Keywords

Health education; Accidental falls; Accident prevention; Education technology: Hospitals

Descriptores

Educación en salud; Accidentes por caídas; Prevención de acidentes; Tecnología educacional; Hospitales

Submetido 17 de Junho de 2020

Aceito

14 de Junho de 202

\section{Autor correspondente \\ Maria Aline Moreira Ximenes} E-mail: aline.ximenes11@hotmail.com

Editor Associado (Avaliação pelos pares): Juliana de Lima Lopes (https://orcid org/0000-0001-6915-6781) Escola Paulista de Enfermagem, Universidade Federal de São Paulo, São Paulo, SP, Brasil

\section{Resumo}

Objetivo: Avaliar a efetividade de intervenção educativa mediada por tecnologia impressa no conhecimento sobre prevenção de quedas em pacientes hospitalizados.

Método: Estudo quase-experimental, do tipo antes e depois, aplicou uma intervenção educativa mediada por cartilha. 0 estudo incluiu 86 pacientes hospitalizados em clínica médica-cirúrgica. Utilizou-se teste de conhecimento sobre prevenção de quedas contendo 12 questões, o qual, foi previamente validado por profissionais com expertise em segurança do paciente. 0 teste de McNemar foi aplicado, para avaliar de forma pareada, as respostas do teste antes e após a intervenção educativa.

Resultados: Observou-se que, antes da intervenção, todas as questões do instrumento tiveram menos acertos. Houve associação entre mudança de conhecimento com: percepção sobre causas de quedas durante a internação $(p=0,008)$; eventos considerados como queda $(p=0,000)$; utilização de meios de apoio à mobilidade $(p=0,000)$; dificuldade de visão e audição como fator de risco ( $p=0,000)$; 0 uso de medicamentos ( $p=0,000)$; importância de informar 0 profissional sobre 0 histórico de quedas $(p=0,007)$; práticas gerais de prevenção $(p=0,000)$; cuidados relacionados ao ambiente $(p=0,000)$ e a prática de exercícios durante a hospitalização $(p=0,000)$.

Conclusão: A intervenção educativa com uso da cartilha foi efetiva quanto a orientação sobre riscos de quedas em pacientes adultos hospitalizados.

\section{Abstract}

Objective: To evaluate the effectiveness of an educational intervention mediated by printed technology on knowledge about fall prevention in hospitalized patients.

Method: This is a quasi-experimental study, of before and after type, applied an educational intervention mediated by a booklet. The study included 86 patients hospitalized in a medical-surgical clinic. A knowledge test on fall prevention was used, containing 12 questions, which was previously validated by professionals with expertise in patient safety. McNemar test was applied to assess, in a paired fashion, test responses before and after the educational intervention.

Results: It was observed that, before the intervention, all questions of the instrument had less success. There was an association between change in knowledge with: perception of causes of falls during hospitalization $(p=0.008)$; events considered as fall $(p=0.000)$; use of means to support mobility $(p=0.000)$; difficulty in seeing and hearing as a risk factor $(p=0.000)$; the use of medications $(p=0.000)$; importance of informing the professional about the history of falls ( $p=0.007)$; general prevention practices $(p=0.000)$; care related to the environment $(p=0.000)$ and the practice of exercises during hospitalization $(p=0.000)$.

'Universidade Federal do Ceará, Fortaleza, CE, Brasi

Universidade da Integração Internacional da Lusofonia Afro-Brasileira, Redenção, CE, Brasil.

3Universidade Estadual Vale do Acaraú, Sobral, CE, Brasil.

4nstituto Federal de Educação, Ciência e Tecnologia de Pernambuco, Pesqueira, PE, Brasil.

Conflitos de interesse: nada a declarar. 
Conclusion: Educational intervention using the booklet was effective in providing guidance on the risk of falls in hospitalized adult patients.

\section{Resumen}

Objetivo: Evaluar la efectividad de una intervención educativa mediada por tecnología impresa sobre os conocimientos en prevención de caídas de pacientes hospitalizados.

Método: Estudio cuasi experimental, tipo antes y después, donde se aplicó una intervención educativa mediante una cartilla. El estudio incluyó 86 pacientes hospitalizados en una clínica médica quirúrgica. Se utilizó una prueba de conocimientos sobre prevención de caídas con 12 preguntas, que fue previamente validada por profesionales especialistas en la seguridad del paciente. Se aplicó la prueba de McNemar para evaluar de forma controlada las respuestas de la prueba antes y después de la intervención educativa.

Resultados: Se observó que, antes de la intervención, todas las respuestas del instrumento tuvieron menos aciertos. Se encontró una relación entre cambios de conocimientos y la percepción sobre causas de caídas durante la internación ( $p=0,008)$, los eventos considerados como caída ( $p=0,000)$, la utilización de medios de apoyo a la movilidad ( $p=0,000)$, las dificultades de visión y audición como factor de riesgo ( $p=0,000)$, el uso de medicamentos ( $p=0,000)$, la importancia de informar al profesional sobre el historial de caídas $(p=0,007)$, las prácticas generales de prevención ( $p=0,000)$, los cuidados relacionados con el ambiente $(p=0,000)$ y la práctica de ejercicios durante la hospitalización $(p=0,000)$.

Conclusión: La intervención educativa con uso de la cartilla fue efectiva respecto a las instrucciones sobre riesgos de caídas en pacientes adultos hospitalizados.

\section{Introdução}

As quedas intra-hospitalares constituem um problema de alta prevalência na segurança do paciente e representam um indicador de qualidade no cuidado em saúde. ${ }^{(1)}$ Dados da Agência Nacional de Vigilância Sanitária (ANVISA) revelam que de janeiro a dezembro de 2017 foram notificadas 8.484 quedas no ambiente hospitalar, e destas, 5,93\% resultaram em óbito. ${ }^{(2)}$ As quedas acarretam repercussóes negativas, como escoriaçóes, lesôes, fraturas e até a morte, comprometem os indicadores de qualidade do serviço, aumentam o tempo de internaçáo e os custos hospitalares. ${ }^{(3)}$ Portanto, é relevante que intervençóes que previnam quedas sejam implementadas, bem como o seu monitoramento, investigação, educação do paciente e familiares. ${ }^{(4)}$

Para subsidiar ações de prevenção de quedas é fundamental que se estabeleça uma abordagem pautada na promoção da saúde do paciente de modo a favorecer sua segurança durante sua estadia no hospital. A educação em saúde como estratégia promotora de saúde proporciona melhorias no conhecimento sobre riscos de quedas, o que pode impactar positivamente na adesão dos pacientes às orientações de prevenção. ${ }^{(5)}$

Destaca-se que a promoção da saúde se caracteriza pela capacitação do indivíduo para evitar fatores condicionantes e determinantes de doenças. No ambiente hospitalar, esta concepção é reafirmada pela Declaração de Budapeste, que ressalta a importância do papel ativo dos pacientes nos serviços de atenção terciária e a melhora da oferta e qualidade dos programas de informação, comunicação e educação em saúde. ${ }^{(5)}$ Portanto, para alcançar a qualidade do cuidado proposta pela Declaração de Budapeste é pertinente que a equipe de enfermagem desenvolva competências específicas no processo de cuidar, favorecendo a assistência em saúde com qualidade.

Essas competências estão descritas no Consenso de Galway, são elas: engajamento eficaz nas práticas de promoção da saúde e educação em saúde como catalisação de mudança, liderança, avaliação das necessidades, planejamento, implementação, avaliação do impacto, defesa de direitos e parcerias. ${ }^{(6)}$ No que se refere às práticas de educação e promoção da saúde, a enfermagem pode utilizar-se de tecnologias como vídeos, jogos, aplicativos e materiais impressos como cartilhas, por serem acessíveis, de baixo custo e propícias para uso em diversos cenários de cuidado, que inclui o hospital. Representam ferramentas viáveis para fortalecer o autocuidado e contribuir para que o aprendizado seja uma experiência mais colaborativa. ${ }^{(7-10)}$

A intervenção educativa com uso de cartilha se respalda nas competências do Consenso de Galway como planejamento, implementação, avaliação e comunicação, permitindo que os pacientes tornem-se ativos na consolidação da competência catalisação de mudanças. ${ }^{(6)}$ Assim, a utilização de tecnologia educacional impressa como ferramenta para educação em saúde sobre prevenção de quedas durante a hospitalização propicia que o momento 
educativo seja mais dinâmico, atrativo e capaz de despertar atenção do paciente para momentos de discussão, resolução de dúvidas, reconhecimento de riscos e estabelecimento de metas. ${ }^{(10)}$ No entanto, existem lacunas no conhecimento sobre a efetividade de cartilhas direcionadas a pacientes hospitalizados. Em busca na literatura científica, observou-se que as tecnologias educacionais para prevenção de quedas concentram-se, principalmente, no enfoque ambulatorial e domiciliar. ${ }^{(7,8,11)}$

Assim, este estudo torna-se relevante por disponibilizar evidências sobre efetividade de ferramenta educativa impressa no cuidado ao paciente hospitalizado. A partir dos resultados deste estudo, a cartilha pode tornar-se instrumento de ensino a ser utilizado em intervençôes educativas sobre prevenção de quedas em diversas instituições hospitalares do Brasil.

Dessa forma, espera-se que os resultados venham colaborar no enfrentamento de eventos adversos que ocorrem nas instituiçóes hospitalares, além de fornecer subsídios aos profissionais de saúde, pesquisadores e formuladores de políticas sobre os benefícios das açóes de educação em saúde para prevenção de quedas mediadas por tecnologias educativas. Ademais, ao avaliar a efetividade dessas ferramentas, é possível fortalecer o papel do enfermeiro como educador e suas competências nas estratégias de promoção da saúde e educação em saúde. Para os pacientes, o uso de materiais impressos pode melhorar a compreensão e adesão às práticas para prevençáo de quedas durante a hospitalizaçáo.

Assim, este estudo tem como objetivo avaliar a efetividade de intervenção educativa mediada por tecnologia impressa no conhecimento sobre prevenção de quedas em pacientes hospitalizados.

\section{Métodos}

Trata-se de estudo quase-experimental do tipo antes e depois.

O cenário do estudo foi a Clínica médica-cirúrgica de hospital referência em trauma, localizado na regiāo Norte do Estado do Ceará, Brasil, no período de agosto a novembro de 2019.
A população-alvo foi constituída por pacientes com 18 anos ou mais, internados na clínica médica-cirúrgica da referida instituição no período de coleta de dados. Como critérios de inclusão considerou-se: estar internado na clínica médica em estudo; ser alfabetizado e obter pontuaçóes mínimas no Mini Exame do Estado Mental (MEEM), foram considerados os pontos de corte: 21 para aqueles com escolaridade entre um e três anos, 24 para indivíduos entre quatro e sete anos de educação formal, e 26 para pessoas com escolaridade acima de oito anos. $^{(12)}$

O MEEM foi utilizado na intenção de certificar que todos os pacientes incluídos possuíam cognição preservada e estavam aptos a compreender a tecnologia educativa impressa, assim como responder as perguntas do teste de conhecimento sobre quedas. Foram excluídos pacientes com instabilidade hemodinâmica, fato que poderia comprometer sua participação na entrevista.

A amostragem ocorreu por conveniência e de forma não probabilística durante os meses de coleta de dados, resultando em 86 pacientes.

Para coleta dos dados, foi utilizado instrumento para caracterização sociodemográfica e teste de conhecimento sobre prevenção de quedas, o qual foi submetido à análise dos juízes. Três enfermeiros pesquisadores foram convidados para verificar a clareza e pertinência do instrumento em avaliar o conhecimento dos pacientes sobre prevenção de quedas.

A seleção dos três juízes foi feita por meio de consulta ao curriculo vitae da plataforma Lattes (CNPq) e por indicação dos pesquisadores, com base na produção bibliográfica e expertise do profissional sobre o tema de interesse. É recomendado que, nesse momento de validação de instrumentos, seja utilizado número ímpar de especialistas bem como, no mínimo três, para avaliação dos itens quanto à equivalência e concordância das respostas. ${ }^{(13)}$

As especialidades dos juízes eram: Juiz 1 Enfermeira, Doutora em Enfermagem e pesquisadora na área de educação em saúde, tecnologias educacionais e segurança do paciente; Juiz 2 Enfermeira, Doutora em Enfermagem e pesquisadora na área de promoção da saúde e segurança do paciente; Juiz 3 - Enfermeiro, Doutor em enferma- 
gem, pesquisador na área de educação em saúde e tecnologias educacionais.

$\mathrm{O}$ instrumento foi adaptado a partir de outro previamente publicado, ${ }^{(14)} \mathrm{o}$ qual foi encaminhado via e-mail aos juízes junto com termo de interesse em participar da pesquisa e formulário de avaliação. Os critérios que os juízes utilizaram para avaliar as questôes foram clareza e pertinência, apresentadas em escala do tipo Likert. Ao lado de cada questão havia espaço para inclusão de possíveis sugestóes. $\mathrm{O}$ coeficiente de validade do conteúdo (CVC) foi considerado aceitável para as questóes que alcançassem o valor mínimo de $0,7 .^{(14)}$

Todas as 12 questóes do instrumento obtiveram CVC acima de 0,88 em relação à clareza e pertinência. Quanto as observaçóes qualitativas, foram realizadas: adequação de linguagem, substituição de palavras complexas por outras mais compreensíveis aos pacientes, inclusão de enunciados verdadeiros e falsos a fim de evitar viés de resposta e redução do tamanho das afirmativas. Todas as sugestóes propostas pelos juízes foram acatadas para que o instrumento pudesse ser utilizado na pesquisa.

Dessa forma, a versão final do instrumento de coleta de dados continha variáveis do perfil sociodemográfico como sexo, idade, ocupação, estado civil, presença de filhos, religiáo, escolaridade e procedência, além das 12 questóes do teste de conhecimento sobre prevenção de quedas no ambiente hospitalar.

A coleta de dados foi realizada por equipe de quatro pesquisadores, composta por uma doutora em enfermagem e três acadêmicos de enfermagem do $10^{\circ}$ semestre, os quais foram previamente treinados para realizar a intervenção educativa de forma padronizada com todos os pacientes. Durante o período de coleta, a equipe se alternava de segunda a sexta-feira, nos turnos manhã e tarde. Ao chegar na clínica médica-cirúrgica, a pesquisadora se apresentava ao enfermeiro do plantão, solicitava a lista de pacientes internados e avaliava quais possuíam quadro clínico estável para participar da pesquisa.

Após essa triagem inicial, os pesquisadores se dirigiam as enfermarias, se apresentavam aos pacientes e informavam os objetivos da pesquisa. Ao manifestar interesse em participar, era verificada a alfabetização e realizada a aplicação do MEEM.
Ao obter pontuação mínima exigida, era solicitada assinatura do Termo de Consentimento Livre e Esclarecido, após a confirmação por escrito, era aplicado instrumento de caracterização sociodemográfica e, em seguida, a avaliação do conhecimento antes da intervenção educativa, por meio do teste de conhecimento, o qual, já foi descrito anteriormente e foi aplicado tanto no pré como no pós-teste.

Após preenchimento do pré-teste, ocorria a intervenção educativa, de forma padronizada, com duração média de 15 minutos em cada paciente. Esse processo ocorreu no ambiente da enfermaria, com o paciente acomodado em seu leito e a pesquisadora em cadeira a beira do leito.

Inicialmente, a pesquisadora introduzia $\mathrm{o}$ tema, abordando questóes sobre a magnitude do problema e a importância da prevenção de quedas. Posteriormente, era disponibilizada a cartilha "Cuidado para não cair nessa", impressa em papel A4, colorida, para leitura individual.

O conteúdo da cartilha inclui os tópicos: "Situaçóes de risco" (apresenta a definição e caracterização de quedas); "Fatores associados a quedas" (contempla os riscos extrínsecos e intrínsecos); "Como evitar complicaçóes" (aborda medidas de prevenção para o paciente e acompanhante); "Ambiente seguro" (possui dicas para manter o ambiente da enfermaria mais seguro); "Exercícios fazem bem" (orientaçóes de exercícios para fortalecimento da musculatura); "Super equipe" (apresenta atribuiçóes da equipe de enfermagem, voltadas à prevençáo de quedas, e as condutas diante de uma situação de queda); "Jogo de sete erros" (lista fatores de risco presentes no ambiente hospitalar). A tecnologia foi construída e validada por profissionais de saúde e avaliada pelo público-alvo como compreensível (IVC=0,98). ${ }^{(10)}$

Enquanto o paciente realizava a leitura, o pesquisador permanecia ao lado do leito para acompanhar todo o processo e realizar quaisquer esclarecimentos em caso de dúvidas. Vale ressaltar que não foi estipulado tempo para concluir a leitura, a fim de evitar pressóes externas e comprometer o processo de aprendizagem.

Com a finalização da leitura, o pesquisador questionava sobre dificuldades de compreensão ou 
qualquer pergunta sobre o tema, a fim de solucioná-las e, em seguida, realizava o pós-teste imediato. Esse procedimento foi utilizado em estudos semelhantes, com aplicação de tecnologias impressas, como cartilhas ${ }^{(15)}$ e audiovisuais, como vídeos. ${ }^{(16)}$

Os dados coletados foram digitados e analisados de acordo com os momentos estabelecidos e compilados no Excel. Posteriormente, foi utilizado o software IBM SPSS Statistics versão 24. O nível de significância adotado foi de $5 \%$ e o intervalo de confiança de $95 \%$. Para avaliar a normalidade dos dados contínuos e definir a escolha do teste (paramétrico ou não paramétrico) foi utilizado o teste de Kolmogorov-Smirnov.

As respostas do teste de conhecimento foram dicotomizadas entre certo e errado. Para comparar a distribuição dos acertos antes e após a intervenção educativa, foi aplicado o teste não paramétrico de McNemar que permite analisar as proporçóes entre as variáveis dicotômicas de forma pareada.

Este estudo foi conduzido em conformidade com a Resolução $n^{\circ}$ 466/2012 tendo sido aprovado pelo Comitê de Ética e Pesquisa da Universidade Estadual Vale do Acaraú (parecer $\mathrm{n}^{\circ}$ 3.377.430/2018) (CAAE 94705218.0.0000.5053). Todos os participantes assinaram o Termo de Consentimento Livre e Esclarecido.

\section{Resultados}

Participaram do estudo 86 pacientes adultos hospitalizados na clínica médica-cirúrgica. A tabela 1 apresenta as características sociodemográficas encontradas.

A maioria dos participantes era do sexo masculino $(62 ; 72,1 \%)$, adultos jovens na faixa etária entre 18 a 24 anos, com idade média de 30 anos ( \pm 12 anos), economicamente ativos (69; 80,2\%), solteiros $(53 ; 61,6 \%)$ e com filhos $(46 ; 53,5 \%)$. A religiáo predominante foi a católica $(55 ; 64,0 \%)$ e a maioria possuía 9 a 11 anos de estudo (46; 53,5\%). Prevaleceram pacientes provenientes da macrorregião de Sobral $(63 ; 73,3 \%)$ devido ao hospital de ensino ser referência em trauma para toda a regiáo norte do estado do Ceará.
Tabela 1. Descrição das características sociodemográficas dos pacientes

\begin{tabular}{lc}
\hline Variáveis & $\mathbf{n}(\%)$ \\
\hline Sexo & \\
$\quad$ Feminino & $24(27,9)$ \\
$\quad$ Masculino & $62(72,1)$ \\
Idade & \\
$\quad 18$ a 24 & $35(40,7)$ \\
$\quad 25$ a 39 & $29(33,7)$ \\
$\quad 40$ a 59 & $19(22,1)$ \\
$\quad 60$ ou mais & $3(3,5)$ \\
Ocupação & \\
$\quad$ Ativa & $69(80,2)$ \\
$\quad$ Inativa & $17(19,8)$ \\
Estado Civil & \\
Casado & $33(38,4)$ \\
Solteiro & $53(61,6)$ \\
Filhos & \\
Sim & $46(53,5)$ \\
Não & $40(46,5)$ \\
Religião & \\
Sem Religião & $15(17,4)$ \\
Católico & $55(64,0)$ \\
Evangélico & $16(18,6)$ \\
Escolaridade (em anos de estudo) & \\
0 - 8 & \\
Procedência & $29(33,7)$ \\
Sobral & $46(53,5)$ \\
Macrorregião & $11(12,8)$ \\
\hline & \\
\hline
\end{tabular}

Muitos pacientes estavam em uso contínuo de medicamentos por via endovenosa (66; 76,7\%). Apenas 30,2\% (26) apresentava comorbidades, com destaque para insônia crônica (9; 34,6\%), Hipertensão Arterial Sistêmica $(6 ; 20,6 \%)$, Diabetes Mellitus (4;15,3\%) e Depressão (4;15,3\%).

O perfil clínico demonstra predomínio de baixo risco para quedas $(42 ; 48,8 \%)$, seguido de médio risco $(31 ; 3,0 \%)$ e alto risco $(13 ; 15,0 \%)$, conforme classificação da escala de Morse. O grau de dependência para realização de atividades de vida diária foi medido pela escala de Katz, que evidenciou independência em 43 (50,0\%) pacientes, dependência parcial em $31(36,0 \%)$ e dependência total em $12(14,0 \%)$. Destaca-se que nenhum participante relatou história prévia de quedas.

$\mathrm{Na}$ tabela 2 são apresentados resultados do teste de conhecimento antes e após a intervenção educativa mediada pela cartilha.

Observou-se que o número de acertos foi menor antes da intervenção educativa em todas as questóes. As que obtiveram melhor significância estatística fo- 
Tabela 2. Comparação dos acertos antes e depois das questões do teste de conhecimento sobre prevenção de quedas

\begin{tabular}{|c|c|c|c|}
\hline Questões & $\begin{array}{c}\text { Acertos antes } \\
\mathrm{n}(\%)\end{array}$ & $\begin{array}{l}\text { Acertos depois } \\
\mathrm{n}(\%)\end{array}$ & $p$-value \\
\hline $\begin{array}{l}\text { Durante a internação podem acontecer quedas quando o paciente vai ao banheiro, quando muda de posição na cama, ou quando anda nos } \\
\text { corredores (Verdadeiro) }\end{array}$ & $78(90,7)$ & $86(100,0)$ & 0,008 \\
\hline $\begin{array}{l}\text { Considera-se como queda quando o paciente é encontrado no chão, ou quando muda de posição e precisa de ajuda, mesmo que não chegue } \\
\text { a cair (Verdadeiro) }\end{array}$ & $50(58,1)$ & $83(96,5)$ & 0,000 \\
\hline Idosos e crianças são grupos com menos risco de quedas (Falso) & $77(89,5)$ & $83(96,5)$ & 0,146 \\
\hline Utilizar muletas ou cadeiras de rodas é um fator de risco para quedas (Verdadeiro) & $38(44,2)$ & $70(81,4)$ & 0,000 \\
\hline Dificuldade em ouvir ou enxergar não contribui para causar quedas em pacientes hospitalizados (Falso) & $68(79,1)$ & $84(97,7)$ & 0,000 \\
\hline Tomar remédios para dormir, para dores fortes, ou depressão podem aumentar as chances de quedas (Verdadeiro) & $63(73,3)$ & $83(96,5)$ & 0,000 \\
\hline $\begin{array}{l}\text { Fatores como o tipo de calçado, pisos com desníveis, banheiro sem piso antiderrapante e cama sem grades são condições de risco para } \\
\text { quedas (Verdadeiro) }\end{array}$ & $83(96,5)$ & $86(100,0)$ & 0,250 \\
\hline As quedas podem gerar problemas aos pacientes e aumentar os dias de internação (Verdadeiro) & $81(94,2)$ & $85(98,8)$ & 0,125 \\
\hline Se você já caiu não deve se preocupar em contar isso a nenhum profissional (Falso) & $73(84,9)$ & $84(97,7)$ & 0,007 \\
\hline $\begin{array}{l}\text { Para evitar quedas é importante só se levantar do leito acompanhado, informar aos profissionais todos os medicamentos que você utiliza e } \\
\text { estar sempre usando seus óculos (Verdadeiro) }\end{array}$ & $69(80,2)$ & $85(98,8)$ & 0,000 \\
\hline $\begin{array}{l}\text { Para um ambiente mais seguro, mantenha as luzes desligadas a noite, bolsas e sacolas devem estar no chão ao lado da maca e os objetos de } \\
\text { uso constante não devem estar próximos ao paciente, pois causam distrações (Falso) }\end{array}$ & $62(72,1)$ & $83(96,5)$ & 0,000 \\
\hline Durante a internação você pode fazer exercícios de alongamento para fortalecer seus músculos e evitar quedas (Verdadeiro) & $71(82,6)$ & $84(97,7)$ & 0,000 \\
\hline
\end{tabular}

${ }^{*}$ Teste de Mc Nemar

ram referentes às causas de quedas durante a internação $(p=0,008)$; quais eventos são considerados como queda $(\mathrm{p}=0,000)$; a utilização de meios de apoio a mobilidade $(\mathrm{p}=0,000)$; dificuldade de visão e audição como fator de risco $(\mathrm{p}=0,000)$; o uso de medicamentos $(\mathrm{p}=0,000)$; importância de alertar sobre o histórico de quedas $(\mathrm{p}=0,007)$; práticas gerais de prevenção $(\mathrm{p}=0,000)$; prevenção relacionada ao ambiente $(\mathrm{p}=0,000)$ e a prática de exercícios durante a hospitalização $(\mathrm{p}=0,000)$. As questóes sobre idosos e crianças como grupos de risco, fatores de risco estruturais e aumento nos dias de internação devido complicações por quedas, não obtiveram diferenças estatísticas, posto que as frequências permaneceram semelhantes nos dois momentos.

\section{Discussão}

Consideram-se como limitaçóes do estudo a exclusão de pacientes analfabetos, com déficit cognitivo e confusão mental, devido ao possível impedimento para leitura individual da cartilha bem como o preenchimento dos instrumentos de coleta de dados. Além disso, a avaliação do conhecimento de forma imediata, sem acompanhamento longitudinal, inviabilizou uma avaliação de médio e longo prazo, e o fato do estudo ter sido realizado somente com pessoas internadas no sistema público, não havendo possibilidade de comparação dos achados com os usuários da rede suplementar de saúde também se configuram como limitações.

Os resultados deste estudo possibilitaram identificar se intervençáo educativa mediada por tecnologia impressa é eficaz na melhora do conhecimento sobre prevenção de quedas em pacientes hospitalizados. Assim, proporciona reflexóes sobre a importância de estratégias de educação em saúde como meio de prevenir quedas em ambiente hospitalar. Além disso, pode subsidiar a prática assistencial ao ofertar ferramenta para prevenção de quedas efetiva e de baixo custo.

Os participantes desse estudo representam população adulta jovem, com média de 30 anos de idade, do sexo masculino e com escolaridade de nove a onze anos de estudo. Pesquisas nacionais e internacionais com escopo similar corroboram com estes achados. ${ }^{(17-21)}$ Vale ressaltar que o nível de escolaridade mais elevado favoreceu a leitura da cartilha e possibilitou melhor compreensão do conteúdo e conclusão do teste de conhecimento sobre quedas.

A classificação da escala de queda de Morse revelou pacientes com baixo e moderado risco. Estudo similar realizado na Paraíba mostrou predomínio de alto risco. ${ }^{(19)}$ Isso avigora a relevância da utilização de instrumentos para predição do risco de quedas, uma vez que o resultado pode estar associado ao setor e características dos pacientes.

A estratégia de educação em saúde utilizada propóe que a adesão do paciente à prevenção de que- 
das pode ser aprimorada quando há conhecimento sobre riscos. Além disso, considera-se que o uso de tecnologias educativas para educação em saúde de pacientes é uma forma de consolidar competências pelo enfermeiro na assistência em saúde, conforme estabelecido no Consenso de Galway. ${ }^{(22)}$

Ao avaliar os resultados em relação ao teste de conhecimento, foi possível observar o incremento do percentual de acertos das questóes quando comparados os momentos pré e pós-intervenção $(\mathrm{p}<0,05)$ nos itens sobre a ocorrência de quedas durante a internaçáo; eventos considerados como queda; a utilização de meios de apoio a mobilidade; dificuldade de visão e audição como fator de risco; o uso de medicamentos; importância de informar os profissionais sobre o histórico de quedas; práticas gerais de prevenção; prevenção relacionada ao ambiente e a realização de exercícios durante a hospitalização.

A percepção sobre ocorrência de quedas durante hospitalização obteve frequência satisfatória de acertos ainda no pré-teste. Após intervenção educativa, todos os participantes revelaram compreender sobre existência de riscos para quedas no ambiente hospitalar. Isso pode ser considerado um bom resultado, pois sugere maior possibilidade de prevenção das quedas pelos pacientes.

$\mathrm{O}$ uso de dispositivos auxiliares como fator de risco para quedas obteve menor número de acertos no pré-teste. $\mathrm{Na}$ Espanha, estudo com 315 pacientes hospitalizados identificou que $42,7 \%$ dos pacientes que utilizavam muletas sem auxílio de acompanhante ou profissional de saúde apresentavam risco elevado para quedas, $27,7 \%$ dos casos sofreram quedas devido à perda de equilíbrio e $22,5 \%$ por tentar realizar a atividade sem auxílio necessário. ${ }^{(23)}$ Logo, o conhecimento destes fatores pelos pacientes pode contribuir para desenvolvimento de estratégias de prevenção voltadas ao controle da mobilidade. ${ }^{(24)}$

Pesquisas observacionais na Ásia e América Latina apontaram que a deficiência auditiva pode ser fator de risco independente para quedas em pacientes hospitalizados. ${ }^{(25,26)}$ Após a intervenção educativa, os pacientes compreenderam sobre necessidade de informar os profissionais sobre déficit visual ou auditivo logo na admissão a fim de contribuir para plano de cuidados específicos mediante necessidade do cliente.

Vale destacar a importância de realizar anamnese e triagem detalhada sobre a função auditiva dos pacientes na admissão, pois, dessa forma, é possível atender com eficácia as necessidades de cuidados com base no grau de deficiência sensorial. Esse processo favorece ainda a sinalização de indivíduos com maiores riscos de quedas devido a condição de saúde, os quais, podem se beneficiar da adaptaçáo do serviço, do plano de cuidados individualizado e de possíveis encaminhamentos dentro da equipe multiprofissional. ${ }^{(27,28)}$

A melhora do conhecimento sobre a associação de déficits de visão e audição com quedas configura-se como resultado importante, uma vez que as quedas são mais frequentes em indivíduos com menor acuidade visual e auditiva, pois estes se tornam menos capazes de identificar e evitar obstáculos.

Quanto ao uso de medicamentos, houve associação na melhora do conhecimento. Observou-se que boa parte dos participantes estavam em uso contínuo de terapia endovenosa, o que os torna susceptíveis aos possíveis efeitos colaterais. Dessa forma, durante a coleta de dados, muitos reconheceram sintomas de hipotensão, tontura e fraqueza, devido ao uso dos medicamentos.

Nesse sentido, os profissionais de enfermagem, por estarem em contato próximo e prolongado com os pacientes, são aptos para identificar riscos relacionados ao uso de classes específicas de medicamentos e fornecer informaçóes sobre eventos adversos associados aos mesmos. Estas estratégias de orientação fortalecem a segurança do paciente durante a internaçáo e podem reduzir danos decorrentes de quedas. ${ }^{(29)}$

Revisão de literatura infere que a ocorrência de quedas em pacientes hospitalizados foi atribuída a ansiolíticos, hipnóticos e sedativos (48\%), antipsicóticos (35\%), opioides e antiepiléticos (30\%) e antidepressivos (22\%). ${ }^{(30)}$ Ao reconhecer os riscos de quedas relacionados a determinados grupos de fármacos os pacientes se tornam ativos no processo de cuidado e parceiros nas intervençóes propostas pelos profissionais de saúde.

Uma questão relevante abordada no teste de conhecimento diz respeito a comunicação com profis- 
sionais. Estudo realizado nos Estados Unidos com 193 pacientes evidenciou que $10 \%$ não pretendiam pedir assistência ao realizar algum comportamento associado ao risco de queda. ${ }^{(31)}$ Logo, a equipe de enfermagem deve deixar claro aos pacientes que eles devem pedir ajuda para se levantar da cama, andar, ir ao banheiro ou sair da cadeira e que isso não é incômodo, é uma atividade para manter sua segurança. ${ }^{(21)}$

Apesar dos participantes não possuírem histórico de quedas, reforça-se a importância de sensibilizá-los a se comunicarem efetivamente com os profissionais, tendo em vista que, os dados aqui apresentados revelam que o conhecimento dos pacientes sobre esta temática pode melhorar a partir da oferta de educação em saúde.

Houve relação importante no conhecimento dos pacientes acerca de complicaçóes decorrentes de quedas após serem abordadas questóes sobre melhor forma de levantar-se da cama, estar sempre acompanhado e cuidados simples com objetos próximos ao leito. Estes fatores de risco associados às quedas são modificáveis e evitáveis. Logo, atitudes voltadas à educação em saúde e prevenção podem interferir positivamente e reduzir as chances de quedas. ${ }^{(11)}$

Quanto a prática de atividade física como forma de prevenção, muitos pacientes consideravam que realizar exercícios durante a hospitalização poderia ofertar riscos ainda maiores para quedas. No entanto, após a leitura do material e esclarecimento de dúvidas, reconheceram os benefícios dos exercícios para mobilidade e fortalecimento da musculatura. Com vistas a reforçar esta prática, na cartilha, são recomendados exercícios de alongamento que podem ser realizados no próprio leito pelo paciente.

Revisão de literatura que analisou orientaçóes clínicas, revisões sistemáticas, metanálises e ensaios clínicos aleatorizados, evidenciou que programas de exercícios para prevenção de quedas não só reduzem taxas de quedas, mas evitam lesóes decorrentes delas. Muitos fatores de risco são corrigíveis por programas de exercícios bem desenhados, como treino de equilíbrio e alongamentos. Dessa forma, devem ser recomendados aos pacientes hospitalizados porque são eficazes na diminuição do número e risco de quedas. ${ }^{(32)}$

Os resultados deste estudo evidenciam que, após intervenção educativa mediada pela cartilha, todos os itens do teste de conhecimento tiveram frequência de acertos superior a $80 \%$. Consolidando este resultado, pesquisa de coorte prospectiva nos Estados Unidos constatou que intervençóes educativas sobre quedas foram associadas a avaliação de conhecimento satisfatória no pós-teste e tiveram relação direta com redução de $22 \%$ nas taxas de quedas no hospital. Da mesma forma, o uso destas intervençóes reduziu $(p<0,001)$ o risco de lesóes por fatores associados à mobilidade, uso do banheiro e cognição prejudicada. ${ }^{(33)}$

Estes resultados relacionam-se com a competência catalisar mudança, definida como possibilidade de modificar comportamentos, bem como empoderar o indivíduo com vistas à melhoria da saúde. ${ }^{(34)}$

Diante dessas evidências, pode-se constatar que a intervenção educativa com uso da cartilha foi responsável pelo aumento significativo no nível de conhecimento dos pacientes sobre riscos de quedas, dos quais, muitos estão expostos durante a hospitalização. Considera-se que o uso de cartilha como tecnologia em saúde é uma ferramenta válida para promoção da saúde e facilitadora do processo educativo em saúde, que oferta contribuiçóes para o conhecimento dos pacientes, família ou profissionais. ${ }^{(35)}$

Os resultados deste estudo fortalecem a importância do papel do enfermeiro como educador em saúde e a necessidade do uso de tecnologias educacionais que facilitem a apreensão do conhecimento pela população-alvo. Dessa forma, acredita-se que a cartilha educativa é efetiva para ser utilizada nos momentos educativos a serem realizados no ambiente hospitalar e que poderá contribuir para o empoderamento dos indivíduos sobre os riscos associados à queda. Destaca-se, ainda, que, no contexto da pandemia de COVID-19, o uso da cartilha como instrumento autodidático deve ser estimulado tendo em vista que o paciente pode realizar a leitura individualmente e aprender sobre prevenção de quedas sem necessitar da presença do enfermeiro neste momento.

Reforça-se, ainda, o caráter inovador do estudo diante da escassez de evidências sobre uso de materiais educativos didáticos voltados aos pacientes adultos hospitalizados sobre prevenção de quedas. Dessa forma, sugere-se novos estudos com conti- 
nuidade do acompanhamento da intervenção e suas implicações na redução da ocorrência de quedas nos serviços de saúde. É imprescindível realização de estudos com intervençóes educativas mediadas por tecnologias que transpassem a dificuldade da comunicação escrita, em face da presença de pacientes não alfabetizados e com baixa escolaridade.

\section{Conclusão}

Intervenção educativa com uso da cartilha foi efetiva quanto à orientação sobre riscos de quedas em pacientes adultos hospitalizados. Assim, este material poderá contribuir para promoção de ambiente hospitalar seguro para o paciente e seu acompanhante, bem como, a melhoria da qualidade da assistência de enfermagem. Além disso, está alinhado aos princípios da promoção da saúde, como autonomia e autocuidado.

\section{Colaborações}

Ximenes MAM contribuiu na concepção do projeto, coleta e interpretação dos dados, redação do artigo e aprovação da versão final a ser publicada. Brandão MGSA, Macêdo TS, Costa MMF e Galindo-Neto NM contribuíram na redação do artigo e aprovação da versão final a ser publicada. Caetano JÁ e Oriá MOB contribuíram na revisão crítica do conteúdo do artigo e aprovação da versão final a ser publicada. Barros LM contribuiu na concepção do projeto, redação do artigo, revisão crítica do conteúdo do artigo e aprovação da versão final a ser publicada.

\section{Referências}

1. de Souza AB, Röhsig V, Maestri RN, Mutlaq MF, Lorenzini E, Alves BM, et al. In hospital falls of a large hospital. BMC Res Notes. 2019;12(1):284.

2. Agência Nacional de Vigilância Sanitária (ANVISA). Boletim Segurança do Paciente e Qualidade em Serviços de Saúde - Incidentes Relacionados à Assistência à Saúde - 2014 a 2018. Brasília (DF): ANVISA; 2018 [citado 2018 Nov 18] Disponível em: https://www20. anvisa.gov.br/segurancadopaciente/index.php/publicacoes/category/ boletins-estatisticos

3. LeLaurin $\mathrm{JH}$, Shorr RI. Preventing falls in hospitalized patients: state of the science. Clin Geriatr Med. 2019;35(2):273-83.
4. Luzia MF, Prates CG, Bombardelli CF, Adorna JB, Moura GM. Characteristics of falls with damage to hospitalized patients. Rev Gaúcha Enferm. 2019;40(Esp):e20180307.

5. Pereira FG, Matias EO, Caetano JÁ, Lima FE. Segurança do paciente e promoção da saúde: uma reflexão emergente. Rev Baiana Enferm. 2015;29(3):271-7.

6. Barry MM, Allegrante JP, Lamarre MC, Auld ME, Taub A. The Galway Consensus Conference: international collaboration on the development of core competencies for health promotion and health education. Glob Health Promot. 2009;16(2):5-11.

7. Hill AM, McPhail SM, Haines TP, Morris ME, Etherton-Beer C, Shorr $\mathrm{R}$, et al. Falls after hospital discharge: a randomized clinical trial of individualized multimodal falls prevention education. J Gerontol A Biol Sci Med Sci. 2019;74(9):1511-7.

8. Imoukhome E, Weeks L, Abidi S. Fall prevention and management App prototype for the elderly and their caregivers: design, implementation, and evaluation. IJEACH. 2020;2(1):48-67.

9. Lima AC, Bezerra KC, Sousa DM, Rocha JF, Oriá MO. Development and validation of a booklet for prevention of vertical HIV transmission. Acta Paul Enferm. 2017;30(2):181-9.

10. Ximenes MA, Fontenele NA, Bastos IB, Macêdo TS, Galindo-Neto NM, Caetano JÁ, et al. Construction and validation of educational booklet content for fall prevention in hospitals. Acta Paul Enferm. 2019;32(4):433-41.

11. Hopewell S, Adedire O, Copsey BJ, Boniface GJ, Sherrington C, Clemson L, et al. Multifactorial and multiple component interventions for preventing falls in older people living in the community. Cochrane Database Syst Rev. 2018;7(7):CD012221.

12. Caramelli $P$, Nitrini R. Como avaliar de forma breve e objetiva o estado mental de um paciente? Rev Assoc Med Bras. 2000;46(4):301.

13. Pasquali L. Instrumentação psicológica: fundamentos e práticas. Porto Alegre: Artmed; 2010.

14. Silveira MB, Saldanha RP, Leite JC, Silva TO, Silva T, Filippin LI. Construction and validation of contente of one instrument to assess falls in the elderly. einstein (São Paulo). 2018;16(2):eA04154.

15. Tsvetanova Dimova R, Dimitrova Dimitrova D, Gencheva Stoyanova R, Angelova Levterova B, Georgiev Atanasov N, Spiridonova Assenova R. The effect of educational intervention on the patient's willingness to carry out the immunochemical faecal occult blood test for colorectal cancer. Zdr Varst. 2015;54(3):230-7.

16. Cowdery JE, Powell JH, Fleming YA, Brown DL. Effectiveness of a short video-based educational intervention on factors related to clinical trial participation in adolescents and young adults: a pre-test/post-test design. Trials. 2019;20(7): 2-7.

17. Severo IM, Kuchenbecker RS, Vieira DF, Lucena AF, Almeida MA. Risk factors for fall occurrence in hospitalized adult patients: a case-control study. Rev Lat Am Enfermagem. 2018;26:e3016.

18. Duarte SC, Stipp MA, Silva MM, Oliveira FT. Adverse events and safety in nursing care. Rev Bras Enferm. 2015;68(1):144-54.

19. Sakai AM, Rossaneis MA, Haddad MC, Vitur DW. Risk of bed falls in adult patients and prevention measures. Rev Enferm UFPE On Line. 2016;10(6): 4720-6.

20. Sato N, Hase N, Osaka A, Sairyo K, Katoh S. Falls among hospitalized patients in an acute care hospital: analyses of incident reports. J Med Invest. 2018;65(1.2):81-84.

21. Abreu HC, Reiners AA, Azevedo RC, Silva AM, Abreu DR, Oliveira AD. Incidence and predicting factors of falls of older inpatients. Rev Saude Publica. 2015;49:37. 
22. Gurgel SS, Ferreira MK, Sandoval LJ, Araújo PR, Galvão MT, Lima FE. Nursing competences in the prevention of falls in children in light of the Galway Consensus. Texto Contexto Enferm. 2017;26(4):e03140016.

23. Santana EC, Moreno CB, Suarez JR, Román CB, Murie-Fernández M. Incidencia de caídas en el hospital de larga-media estancia. Factores de riesgo y estrategias para la prevención. Neurología. 2019;1296:16.

24. Alves EF, Bezerra PP. Fatores associados ao uso de cadeira de rodas por idosos institucionalizados. Cien Saude Colet. 2017;22(11):360714.

25. Kasuga T, Aruga F, Ono K, Hiratsuka Y, Murakami A. Visual impairment as an independent risk factor for falls in hospitalized patients. Can J Ophthalmol. 2017;52(6):559-63.

26. Rosa VP, Cappellari FC, Urbanetto JS. Analysis of risk factors for falls among institutionalized elderly persons. Rev Bras Geriatr Gerontol. 2019;22(1):e180138.

27. Davidson JG, Guthrie DM. Older adults with a combination of vision and hearing impairment experience higher rates of cognitive impairment, functional dependence, and worse outcomes across a set of quality indicators. J Aging Health. 2019;31(1):85-108.

28. Araújo JN, Fernandes AP, Moura LA, Santos MM, Ferreira Júnior MA, Vitor AF. Validation of nursing outcome content fall prevention behavior in a hospital environment. Rev Rene. 2017;18(3):337-44.
29. Duarte SC, Azevedo SS, Muinck GC, Costa TF, Cardoso MM, Moraes $J R$. Best safety practices in nursing care in neonatal intensive therapy. Rev Bras Enferm. 2020;73(2):e20180482.

30. Ribeiro TB, Melo DO, Maia F0, Ribeiro E. Medication-related inpatient falls: a critical review. Braz J Pharm Sci. 2018;54 (1):e17355.

31. King B, Pecanac K, Krupp A, Liebzeit D, Mahoney J. Impact of fall prevention on nurses and care of fall risk patients. Gerontologist. 2018;58(2):331-40.

32. Cunha P, Pinheiro LC. 0 papel do exercício físico na prevenção das quedas nos idosos: uma revisão baseada na evidência. Rev Port Med Geral Fam. 2016;32(2):96-100.

33. Titler MG, Conlon P, Reynolds MA, Ripley R, Tsodikov A, Wilson DS, et al. The effect of a translating research into practice intervention to promote use of evidence-based fall prevention interventions in hospitalized adults: a prospective pre-post implementation study in the U.S. Appl Nurs Res. 2016;31:52-9.

34. Allegrante JP, Barry MM, Airhihenbuwa CO, Auld ME, Collins JL, Lamarre $\mathrm{MC}$, et al. Domains of core competency, standards, and quality assurance for building global capacity in health promotion: the galway consensus conference statement. Health Educ Behav. 2009;36(3):476-82.

35. Paula FM, Beserra NC, Lopes RC, Guerra DR. Elaboration of didactic material for processing health products in primary health care units. Rev SOBECC. 2017;22(3):165-70. 